This is a pre-copy-editing, author-produced PDF of an article accepted for publication in Schizophrenia Bulletin following peer review. The definitive publisher-

authenticated version Dominguez, M. d. G., Wichers, M., Lieb, R., Wittchen, H.-U. \& van Os, J. (2011). Evidence That Onset of Clinical Psychosis Is an Outcome of Progressively More Persistent Subclinical Psychotic Experiences: An 8-Year Cohort Study. Schizophrenia Bulletin, 37(1), 84-93 is available online at:

http://schizophreniabulletin.oxfordjournals.org/content/37/1.toc. 


\title{
Evidence That Onset of Clinical Psychosis Is an Outcome of Progressively More Persistent Subclinical Psychotic Experiences: An 8-Year Cohort Study
}

\author{
M.D.G. Dominguez ${ }^{2}$, Marieke Wichers ${ }^{2}$, Roselind Lieb ${ }^{3,4}$, Hans-Ulrich Wittchen ${ }^{3,5}$, and Jim \\ van $\mathrm{Os}^{1,2,6}$ \\ ${ }^{1}$ To whom correspondence should be addressed; tel: p31-43-3875443, fax: p31-43-3875444, e-mail: \\ j.vanos@sp.unimaas.nl \\ ${ }^{2}$ Department of Psychiatry and Neuropsychology, South Limburg Mental Health Research and Teaching \\ Network, EURON, Maastricht University Medical Centre, PO BOX 616 (DRT 10), 6200 MD Maastricht, The \\ Netherlands; \\ ${ }^{3}$ Clinical Psychology and Epidemiology Unit, Max Planck Institute of Psychiatry, Kraepelinstrasse 2, Munich, \\ Germany; \\ ${ }^{4}$ Epidemiology and Health Psychology, Institute of Psychology, University of Basel, Missionsstrasse 60-62, \\ Basel, Switzerland; \\ ${ }^{5}$ Institute of Clinical Psychology and Psychotherapy, Technical University Dresden, Chemnitzerstr 46, Dresden, \\ Germany; \\ ${ }^{6}$ Division of Psychological Medicine, Institute of Psychiatry, De Crespigny Park, Denmark Hill, London, UK
}

This study examined the hypothesis that developmental expression of psychometric risk in the form of subclinical psychotic experiences in the general population is usually transitory but in some instances may become abnormally persistent and progress to a clinical psychotic state. A prospective cohort study was conducted in a general population sample of 845 adolescents, aged 14-17 years, in Munich, Germany (Early Developmental Stages of Psychopathology Study). Expression of psychosis was assessed 4 times (T0-T3) over a period of 8.4 years. Transition from subclinical psychosis at T0-T2 to clinical psychosis in terms of impairment at T3 was examined as a function of the level of prior persistence of subclinical psychosis (present never, once, twice, or thrice). The more the subclinical psychosis persisted over the period T0-T2, the greater the risk of transition to clinical psychosis at T3 in a dose-response fashion (subclinical psychosis expression once over T0-T2: odds ratio [OR] 51.5 [95\% confidence interval \{CI\} 5 0.6-3.7], posttest probability [PP] 5 5\%; twice: OR 55.0 [95\% CI51.6-15.9], PP 5 16\%; at all 3 measurements: OR 59.9 [95\% CI 5 2.5-39.8], PP 5 27\%). Of all clinical psychosis at T3, more than a third (38.3\%) was preceded by subclinical psychotic experiences at least once and a fifth (19.6\%) at least twice. Consequently, a significant proportion of psychotic disorder may be conceptualized as the rare poor outcome of a common developmental phenotype characterized by persistence of psychometrically detectable subclinical psychotic experiences. This may be summarized descriptively as a psychosis proneness-persistence-impairment model of psychotic disorder.

Key words: epidemiology, general population, psychosis onset, adolescence, transition to clinical relevance, subclinical psychosis 


\section{Introduction}

Psychosis is commonly considered a rare phenomenon outside the range of normal human experience, giving rise to significant disability. However, the type of distribution expected for disorders of multifactorial interactive etiologies is continuous, ${ }^{1,2}$ and many mental disorders have accordingly been shown to exist along a spectrum including subclinical expressions, evidenced by, eg, relatively high rates of expression of bipolar spectrum symptoms, subthreshold depressive states, and social restriction associated with autism. ${ }^{3-6}$

Similar findings have been reported for psychosis. ${ }^{1}$ The clinical psychosis phenotype has not only been shown to be much less rare than previously thought ${ }^{7}$ but is also thought to be expressed at levels well below its clinical manifestation. ${ }^{8-11}$ Apart from strong evidence of phenomenological continuity in form and structure, ${ }^{1}$ continuity between clinical and subclinical phenotypes is further suggested by (1) similar associations with demographic factors, in particular the negative association with age and the positive association with single marital status and social disadvantage, ${ }^{1,10,12,13}$ (2) transitions over time from the subclinical to the clinical, ${ }^{8,14,15}$ (3) familial co-clustering of the clinical and the subclinical, ${ }^{16}$ (4) strong dose-response effects of the urban environment on both phenotypes, ${ }^{17}$ (5) sharing of cognitive and motor deficits, ${ }^{18,19}$ and (6) sharing of risk genes ${ }^{20}$ and of environmental risk factors such as cannabis. ${ }^{21}$

Many questions remain, however, with regard to the hypothesized continuity over time from subclinical to clinical psychotic states. The seminal study by Poulton et al ${ }^{14}$ demonstrated that more than $25 \%$ of participants with low-grade psychotic experiences at age 11 years developed a clinical psychotic disorder by age 26 years. However, an equally important conclusion of the study was that the great majority of children with expression of psychotic experiences would never develop a psychotic disorder - psychosis was only a transitory developmental state for most. Similar conclusions were reached by other, less lengthy followup studies in adult population cohorts, with transition rates of around $10 \% .{ }^{8,15}$ Cougnard et $\mathrm{al}^{22}$ recently suggested that one of the reasons why most developmental expression of psychosis remains transitory is that exposure to additional environmental risks over development, such as cannabis use, childhood trauma, and urban environment, is necessary in order for subclinical psychosis to first become persistent and, second, to deteriorate into a clinical psychotic state (figure 1). However, to date, no evidence has been presented suggesting that abnormal persistence of a common and normally transitory developmental subclinical psychosis phenotype is the precursor state of clinical deterioration.

In the current article, it was examined whether subclinical psychotic experiences with a greater tendency to persist over time would have a greater probability to become clinically relevant in terms of dysfunction and need for care (impairment). The hypothesis of greater persistence resulting in greater probability of transition from subclinical to clinical psychosis characterized by impairment was tested using a prospective, observational study in a large, general population sample of adolescents followed over a period of more than 8 years.

\section{Method}

\section{Sample}

Data came from the Early Developmental Stages of Psychopathology (EDSP) Study, which collected data on the prevalence, incidence, risk factors, comorbidity, and course of mental disorders in a random, representative population sample of adolescents and young adults in 
the general population. The baseline sample, following ethics committee approval, was randomly drawn, in 1994, from the respective population registry offices of Munich and its 29 counties, to mirror the distribution of individuals expected to be 14-24 years of age at the time of the baseline (T0) interview in 1995. The base population were all those born between June 1, 1970, and May 31, 1981, registered as residents in these localities and having German citizenship. These registers can be regarded as highly accurate because (1) each German is registered by his town, (2) they are regularly updated, (3) in the interest of scientific studies, any number of randomly drawn addresses with a given sex and age-group can be obtained, and (4) strict enforcement of registration by law and the police applies. More details on the sampling, representativeness, instruments, procedures, and statistical methods of the EDSP Study sample have previously been presented. ${ }^{23,24}$

\section{Study Design}

The overall design of this cohort study is longitudinal and prospective, consisting of a baseline (T0) and 3 follow-up surveys, covering a time period of on average 1.6 years (T0$\mathrm{T} 1, \mathrm{SD}=0.2)$, 3.5 years $(\mathrm{T} 0-\mathrm{T} 2, \mathrm{SD}=0.3)$, and 8.4 years $(\mathrm{T} 0-\mathrm{T} 3$, range $=7.3-10.5 \mathrm{y}, \mathrm{SD}=$ 0.7). Because the primary goal of the study was to examine the incidence and developmental risk factors for psychopathology, the younger group (14-15 y), presumed to have the highest incidence density, was sampled at twice the rate of persons aged 16-21 years, and the oldest group (22-24 y) was sampled at half this rate. For the same reason, subjects aged 14-17 years at baseline were examined at the 4 time points, and subjects aged 18-24 years were assessed only 3 times.

The present study is based on a subset of EDSP Study respondents, namely, the younger cohort (aged 14-17 $\mathrm{y}$ at baseline) only ( $\mathrm{T0}, \mathrm{n}=1395$, response rate $=75 \%$ ), thus ensuring an adolescent population at risk of developing psychotic experiences and subsequent transition to clinically relevant psychotic states over 4 assessment periods. Response rates for the younger cohort were respectively $88 \%$ at T1 $(n=1228), 83 \%$ at T2 $(n=1169)$, and $73 \%$ at T3 $(n=$ 1022). After description of the study to the subjects, written informed consent was obtained.

\section{Instruments}

The Self-report Symptom Checklist-90-R. At all time points, participants completed the selfreport symptom checklist-90-R (SCL-90-R), a comprehensive self-report symptom inventory, multidimensional in nature, and oriented to screen for a broad range of psychological problems and psychopathology in community respondents and respondents with somatic and psychiatric disorders. It contains 90 items, scored on a 5-point severity scale, measuring 9 primary symptom dimensions named „somatization,“ „obsessive-compulsive,“ „interpersonal sensitivity,“ „depression,“ „anxiety,“ „hostility,“ „phobic anxiety,“ „paranoid ideation,“ and „psychoticism.“

Reliability and validity of the SCL-90-R were established previously. ${ }^{25}$ The time frame is the past 2 weeks.

The Munich-Composite International Diagnostic Interview. Participants were also assessed using the computerized version of the Munich-Composite International Diagnostic Interview (DIA-X/M-CIDI), ${ }^{26}$ an updated version of the World Health Organization's CIDI version 1.2. ${ }^{27}$ The DIA-X/M-CIDI is a comprehensive, fully standardized diagnostic interview and assesses symptoms, syndromes, and diagnoses of various mental disorders in accordance with the definitions and criteria of the International Classification of Diseases, Tenth Revision, and 
Diagnostic and Statistical Manual of Mental Disorders (Fourth Edition), along with information about onset, duration, severity of symptoms, and psychosocial impairment. The CIDI has been primarily designed for use in epidemiological studies of mental disorders and can also be used for clinical purposes. It is divided into 16 sections: 1 sociodemographic section, 12 sections assessing 288 symptoms of groups of mental disorders (including „,somatoform and dissociative,“ „phobic and other anxiety,“ „depressive and dysthymic,“ „manic and bipolar affective,“ „schizophrenia and other psychotic,“ „eating,“ „dementia and other cognitive,“ „posttraumatic stress,“ as well as „tobacco,“ „alcohol,“ and „,substancerelated“ disorders), and 3 final sections containing concluding questions, interviewer observations, and interviewer ratings.

The instrument, designed for use by trained interviewers who are not clinicians, has shown high interrater $(\mathrm{j}>0.70)^{28,29}$ and test-retest reliability. ${ }^{30,31}$ However, the assessment of psychosis with CIDI by lay interviewers is not considered reliable. ${ }^{30,32}$ Therefore, in the EDSP Study, trained and experienced clinical interviewers at the level of psychologist, who were allowed to probe with follow-up clinical questions, conducted the interviews in the respondents' homes.

At baseline, the DIA-X/M-CIDI lifetime version was used. At each of the follow-up assessments, participants applied the interval version, which covers the period of assessment from the last interview until the next. However, data on the $\mathrm{G}$ section concerning psychosis and its clinical relevance were only collected at time point 2 (lifetime version) and time point 3 (interval version).

\section{Construction of SCL-Psychosis Subscale}

The 2 symptoms dimensions relevant for psychosis of the SCL-90-R, the so-called paranoid ideation and psychoticism subscales, were used to assess persistence of psychotic experiences over the period T0-T3. The 2 subscales include self-reports on psychotic experiences that can be regarded, if not as clear-cut psychotic symptoms, as a subclinical expression of psychotic experiences. The 6-item paranoid ideation subscale represents paranoid behavior fundamentally as a disordered mode of thinking, including the cardinal characteristics of projective thought, hostility, suspiciousness, grandiosity, centrality, and fear of loss of autonomy (items 8, 18, 43, 68, 76, and 83). The 10-item psychoticism subscale was developed in a fashion to represent the construct as a continuous dimension of human experience, including items indicative of a withdrawn, isolated, schizoid lifestyle, and first-rank symptoms of schizophrenia, such as hallucinations and thought broadcasting (items 7, 16, 35, $68,77,84,85,87,88$, and 90$)$. The severity scale of each symptom was scored from „not at all“ (0) to „extremely“ (4). The psychoticism and paranoia scales are strongly correlated (in the current sample, mean correlation over T0-T3 was .7), and the items in the scales have high predictive validity for future deficiency in social achievement and functioning. ${ }^{33}$ Therefore, conform previous research in this sample, ${ }^{17,34}$ the psychoticism and paranoid ideation subscales were combined into one Psychosis scale (hereafter „SCL-psychosis subscale“) by summing their scores. The resulting SCL-psychosis subscale thus was a continuous score displaying a half-normal distribution at all time points (T0-T3). Previous research has shown that instruments assessing psychotic experiences dimensionally typically display a half-normal distribution. ${ }^{2}$ 
In order to examine the hypothesis relating to persistence over time, a discrete variable indicating presence or absence of psychosis across interview waves was, per definition, necessary. For the purpose of the analyses, a dichotomous psychosis variable was created using a cutoff point to define the group of individuals with the highest $10 \%$ of scores of the SCL-psychosis subscale (hereafter „psychosis expression“), consistent with previous analyses in this sample $\mathrm{e}^{22,34}$ and congruent with the meta-analytic rate for the prevalence of psychotic symptoms in the general population. ${ }^{2}$ Nevertheless, as a $10 \%$ cutoff remains arbitrary, sensitivity analyses were conducted using more and less conservative cutoff point values of the highest $5 \%$ and the highest $15 \%$ of the SCL-psychosis subscale.

A variable was defined indicating the degree of persistence of psychotic experiences over the period T0-T2. This resulted in a summary score variable consisting of 4 levels of psychosis expression: never (0), once (sporadic) (1), twice (recurrent) (2), and thrice (persistent) (3). This variable will hereafter be referred to as „psychosis persistence.“

\section{Assessment of Clinical Relevance of Psychotic Experiences at T2 and T3}

Clinical relevance related to psychotic experiences was assessed by interview ratings from the DIA-X/M-CIDI in terms of psychotic impairment, representing the first step that needs explaining and predicting in tracking the ontogenesis of psychotic disorder in the general population. In order to define psychotic impairment, secondary dysfunction and help-seeking behavior were only examined in individuals with evidence of DIA-X/MCIDI-positive psychotic symptoms at T2 (lifetime) and T3 (interval version).

Presence of positive psychotic experiences was broadly defined as any rating of „present“ on any of the 20 DIAX/ M-CIDI core psychosis items (G1, G2a, G3-G5, G7-G13, G13b, G14, G17, G18, G20, G20C, G21, and G22a), including 14 delusion items, 5 hallucination items, and 1 item on passivity phenomena. Items relate to classic psychotic symptoms involving, eg, persecution, thought interference, auditory hallucinations, and passivity phenomena. Participants were first invited by the psychologist to read a list of all the psychotic experiences and then asked whether they ever experienced such symptoms (list and phrasing available upon request). All these psychosis items were rated in 2 ways: absent (1) and present (5); all the psychosis DIA-X/M-CIDI items used in the present study were coded in a dichotomous manner without intermediate levels.

Help Seeking. Help-seeking behavior secondary to psychotic experiences was assessed using 3 DIA-X/M-CIDI items. Two psychosis section items were used: G16 (delusions) and G23 (hallucinations), which were phrased as follows: „Did you tell a doctor about ... (the psychosis section beliefs/experiences previously acknowledged by the participant along with a visual representation from the response booklet) you have had?" A third item from the concluding section was added (Q1DG); participants were shown a list on which several types of outpatient or inpatient institutions for mental health problems were mentioned, ranging from general practitioner or school psychologist to psychiatric sheltered housing, and asked whether they had ever sought help at any of these institutions because of psychotic symptoms as elicited in the DIA-X/M-CIDI G section. The 3 items were rated in a dichotomous manner: no (1) and yes (5). Using these 3 help-seeking behavior items, a dichotomous variable „help seeking" was constructed, indicating a positive answer on any of the 3 questions (value label: 1) vs negative answers on all 3 questions (value label: 0 ).

Dysfunction. The dysfunction score of the psychosis DIA-X/M-CIDI section assessed the effect of the psychotic experiences on (1) feeling upset, unable to work, go to places, or enjoy 
oneself, at the time of having these experiences (item G28), (2) being less able to work since these experiences began (item G29), (3) being less able to make friends or enjoy social relationships since these experiences began (item G29a), and (4) how much their life and everyday activities were impaired when these experiences were at their worst (item G36). These 4 items were rated in a dichotomous manner: no (1) and yes (5).A dichotomous variable „dysfunction“ was dichotomously constructed, representing a positive answer on any of the 4 questions (value label: 1 ) vs negative answers on all 4 questions (value label: 0 ).

The Outcome Measure: Psychotic Impairment. Clinically relevant psychosis was finally defined in terms of „psychotic impairment,“ a variable created as a combined outcome of help seeking and dysfunction. This was rated as „,0 in subjects with psychotic experiences who scored „0“ on both help seeking and dysfunction and „1“ in subjects scoring „1“ on either or both help seeking and dysfunction.

Caseness. In order to validate the outcome measure psychotic impairment in terms of clinical relevance, the X16 DIA-X/M-CIDI item was used. This item, rating the interviewer's opinion regarding clinical evidence of psychological ill health, consists of 4 levels: essentially not noticeable (0), not very noticeable (1), clearly ill (2), and very ill (3). The dichotomous variable „caseness“ was defined as any score above 1 , thus indicating individuals with a noticeable level of psychiatric caseness.

\section{Risk Set}

All the analyses were conducted in the younger cohort who (1) had been examined in the 4 waves of the study, (2) had completed the psychoticism and paranoia SCL-90-R subscales at T0-T3, and (3) had completed the DIA-X/M-CIDI psychosis G section at T2 and T3 (n = 845). Of the 1395 individuals in the younger cohort at baseline, 845 fulfilled the risk set criteria.

Of the 1022 participants belonging to the younger cohort that completed the 4 waves, 94 individuals did not complete the self-report SCL-90-R at T1, 65 at T2, and 13 at T3. In addition, the $\mathrm{G}$ section of the DIA-X/M-CIDI was not available for 5 participants at T2. This resulted in a risk set of 845 participants who were included in the analysis.

Thus, a risk set of 845 participants remained from the total number of 1395 participants that composed the younger cohort at baseline. Of the 550 individuals not included in the risk set (hereafter: „nonincluded group“), the reason was loss to follow-up over the 8-year period in 373 and partial nonresponse in 177.

\section{Statistical Analyses}

All analyses were conducted using the software package STATA, version 9.2 (Stata Corporation, College Station: TX; 2006).

In order to examine representativeness, the risk set $(n=845)$ was compared with the nonincluded group $(n=550)$ in terms of sociodemographic characteristics such as age, gender, level of education, social status, and urban residence.

The focus of the study was on psychosis persistence in adolescents over a period of approximately 3.5 years (T0-T2), integrating the scores of 3 waves (T0, T1, and T2) and analyzing the influence of the degree of persistence on the onset of psychotic impairment at 
T3 (figure 1). To this end, logistic regression analyses were performed to examine the associations between T0-T2 psychosis persistence on the one hand and T3 psychotic impairment on the other. Associations were expressed as odds ratios (ORs) and their 95\% confidence intervals (CIs). In order to ensure that the analyses focused on true new onsets of psychotic impairment at T3, any individual with psychotic impairment assessed lifetime at T2 was excluded.

In order to calculate posttest probabilities (PPs) for T0-T2 psychosis persistence in relation to the T3 outcome of psychotic impairment, excluding any individual with lifetime T2 psychotic impairment, the STATA DIAGTEST procedure was used.

For both the logistic regression analyses and the calculation of PPs, a further check was introduced by additionally excluding individuals reporting at $\mathrm{T} 2$ that they had received any professional help for any psychological problem (DIA-X/M-CIDI Q1 item).

In addition, sensitivity analyses were conducted using more and less conservative cutoff point values of the highest $5 \%$ and the highest $15 \%$ of the SCL-psychosis subscale. Logistic regression was used to validate the T3 outcome measure psychosis impairment against the T3 caseness variable.

\section{Results}

\section{Subject Characteristics}

The average age of the risk set at T0 was 15.08 years (range $=14-17$; SD $=1.1$ ). Of all participants, 444 were boys (52.5\%) and 401 girls (47.5\%). Other demographic characteristics are depicted in table 1.

In comparison with the risk set $(n=845)$, individuals from the nonincluded group $(n=550)$ were of similar age (mean age $=15.18 \mathrm{y}$ ), gender distribution (boys: 49.1\%), or urban residence (69.1\% vs $72 \%$ for included vs nonincluded, respectively). The nonincluded group had a lower level of education (included lower level: 9.7\% vs nonincluded lower level: $28.6 \%$; v2 $=100.41, \mathrm{P}=.000$ ) and lower social status (included lower status: $3.4 \%$ vs nonincluded lower status: $6.6 \%$; $2=12.36, \mathrm{P}=.006$ ).

\section{Occurrence of Psychotic Experiences}

The mean score of the continuous SCL-psychosis subscale was $21.5(\mathrm{SD}=5.6)$ at T0, 19.5 $(\mathrm{SD}=4.9)$ at $\mathrm{T} 1,19.5(\mathrm{SD}=4.7)$ at $\mathrm{T} 2$, and $18.7(\mathrm{SD}=4.7)$ at $\mathrm{T} 3$. With the interview ratings of the DIA-X/M-CIDI, the lifetime prevalence of psychotic symptoms at T2 was $21.8 \%(\mathrm{n}=$ 184), and the interval cumulative incidence of psychotic symptoms at T3 was $12.7 \%(\mathrm{n}=$ 107).

The distribution of the SCL-90-R psychosis persistence variable over T0-T2 was never: 78.9\% ( $n=666)$, once (sporadic): $15.6 \%(n=132)$, twice (recurrent): $3.9 \%(n=33)$, and thrice (persistent): $1.7 \%(n=14)$. Of the 83 individuals with psychosis expression at T0, 32 (38.6\%) again had psychosis expression at either T1 or T2. Of the 72 individuals with psychosis expression at T1, 29 (40.3\%) had psychosis expression at T2. Based on the DIA$\mathrm{X} / \mathrm{M}$-CIDI interview ratings, of the 184 (21.8\%) individuals with any DIA-X/M-CIDI psychotic symptom at T2, 51 (27.7\%) again had evidence of psychotic symptoms at DIA$\mathrm{X} / \mathrm{M}$-CIDI interview at T3. 


\section{Occurrence of Psychotic Impairment}

Of the 184 individuals with lifetime presence of DIA-X/ M-CIDI psychotic symptoms at T2, 64 (34.8\%) had evidence of psychotic impairment. Of the 107 individuals with interval presence of DIA-X/M-CIDI psychotic symptoms at T3, 45 (42.1\%) had evidence of psychotic impairment.

Of the 64 individuals with psychotic impairment at T2, 11 (17.2\%) had again evidence of psychotic impairment at T3.

\section{Proportion of T3 Psychotic Impairment With Prior T0-T2 Psychosis Expression}

In terms of attributable risk, of the 47 individuals who displayed T3 psychotic impairment, 18 (38.3\%) had evidence of psychosis expression at any time point between T0 and T2, of which $9(19.6 \%)$ more than once.

\section{Transition to T3 Psychotic Impairment}

There was a strong association between T3 psychotic impairment and the T3 clinical caseness variable $(\mathrm{OR}=9.4,95 \% \mathrm{CI}=3.1-28.6)$

The absolute rate and relative risk of onset of T3 psychotic impairment as a function of the degree of T0-T2 psychosis persistence are depicted in table 2. Excluding those with lifetime evidence of psychotic impairment at T2, the rates of T3 transition to psychotic impairment at the 4 levels of psychosis persistence (from 0 to 3$)$ were $3.7 \%(n=23), 5.3 \%(n=6), 16.0 \%(n$ $=4)$, and $27.3 \%(\mathrm{n}=3)$, yielding ORs (levels $1-3$ relative to 0$)$ of $1.5(95 \% \mathrm{CI}=0.6-3.7 ; \mathrm{p}=$ $.414)$, 5.0 (95\% CI = 1.6-15.9; $\mathrm{p}=.006)$, and 9.9 (95\% CI = 2.5-39.8; $\mathrm{p}=.001)$, respectively. Additional exclusion of those with $\mathrm{T} 2$ receipt of professional help for mental ill health similarly did not change these results (ORs of 1.1 [95\% CI $=0.4-3.2 ; \mathrm{p}=.893$ ], 3.1 [95\% CI $=0.7-14.0 ; \mathrm{p}=.151]$, and 16.5 [95\% CI = 3.7-73.6; $\mathrm{p}=.000]$, respectively).

Sensitivity analyses, using more and less conservative cutoff values for the SCL-psychosis subscale, showed the same pattern of increase in the rates of T3 transition to psychotic impairment. Thus, for the more conservative 5\% cutoff, risk of transition increased from the sporadic $(\mathrm{OR}=2.8,95 \% \mathrm{CI}=1.1-7.1)$ to the persistence level $(\mathrm{OR}=12.6,95 \% \mathrm{CI}=1.1-$ 143.2). For the less conservative $15 \%$ cutoff, risk for transition increased similarly from the sporadic $(\mathrm{OR}=1.6,95 \% \mathrm{CI}=0.7-3.7)$ to the persistence level $(\mathrm{OR}=7.5,95 \% \mathrm{CI}=2.3-$ 24.8).

The PPs for T0-T2 psychosis persistence in relation to T3 psychotic impairment, excluding individuals with lifetime evidence of T2 psychotic impairment, increased progressively from the sporadic ( $\mathrm{PP}=5.3 \%$; 95\% CI $=3.7 \%-6.9 \%)$ to the persistence level $(\mathrm{PP}=27.3 \% ; 95 \%$ $\mathrm{CI}=23.8 \%-30.7 \%$ ) (table 2). Predictive values were even higher when the risk set was additionally restricted to individuals with no T2 receipt of professional help for mental health problems (table 2). 


\section{Discussion}

\section{Findings}

This is the first study examining the influence of persistence of subclinical psychotic experiences in a sample of adolescents and young adults in the general population in relation to the transition to clinically relevant psychosis. The results showed, first, that subclinical psychosis was confirmed to be common (psychosis expression rates of 22\%) and mostly transitory, recurrence or persistence occurring in 30\%-40\% of cases. Second, the findings demonstrated that persistent positive symptoms at a subclinical level pose a significant risk and can be considered a marker of emerging clinical states. A dose-response relationship was found: the longer the period over which subclinical psychosis remained abnormally nontransitory, the greater the risk of developing associated impairment. Third, although most subclinical psychosis was transitory, these results showed that the impact of the relationship between the clinical and the subclinical was not negligible: Around two-fifths of new onset, clinically relevant psychosis in young adults could be traced to the subclinical psychosis phenotype expressed up to 8 years earlier, and one-fifth had displayed evidence of a recurrent or persistent subclinical phenotype. For some individuals, psychosis is thus the denouement of a long process of subclinical experiences and functional deficits. Consequently, these findings provide evidence for the continuity over time from subclinical persistent psychotic experiences to clinical psychotic states, yielding insight into the dynamics preceding the clinical onset of psychotic disorder from a population perspective.

\section{Persistence of Subclinical Psychosis Expression}

The recurrence-persistence rate of subclinical psychosis in this study was higher than reported in previous population- based studies. In a 2-year follow-up of a general population sample aged 18-64 years, Hanssen et al $^{15}$ found a 2-year $8 \%$ persistence rate of baseline subclinical psychosis and an $8 \%$ transition rate from subclinical to clinical psychosis. In the present study, the recurrence-persistence rate was 30\%-40\% over time, considerably higher. The most likely factor to explain this difference is the much younger age of the current sample. Given the rapid decline of psychosis expression with age, ${ }^{10,12,13}$ continuity over time will become less likely with advancing age. Thus, in a previous follow-up study of adolescents, a 3 -year persistence rate was found to be $40 \%$, similar to that of the current study. ${ }^{35}$

\section{The Persistent Subclinical Psychosis Pathway Into Clinical Psychosis}

Although the development of subclinical psychotic symptoms is an initial step to the emergence of psychotic disorder in a significant proportion of cases, not more than two-fifths of all psychotic impairment could be attributed to this pathway. This likely represents an underestimate, however, caused in part by the uneven sampling intervals from T0 to T3. Thus, the relatively wide sampling interval between T2 and T3 (4.9 y on average) may have resulted in participants underreporting experiences and instances of help seeking. Similarly, the SCL90-R assesses symptoms over the last 2 weeks, thus creating the possibility of underreporting psychotic experiences over the period T0-T2. In addition, other domains of psychopathology, such as depression and anxiety, may also contribute to prediction and attributable risk and need to be investigated further. ${ }^{36}$ Furthermore, more work is needed on the role of cognitive alterations in the ontogenesis of psychotic states, given the fact that meta-analytic work indicates that this domain is orthogonal to the positive and affective symptoms of psychosis, ${ }^{37}$ yet is likely to impact on the domain of functional impairment in the onset of clinical psychotic states. 
Even if it represents a likely underestimation, the fact that $40 \%$ of new onset, clinically relevant psychosis can be traced to the early subclinical psychosis phenotype in the general population sheds light on the ontogenesis of psychotic states in the general population. However, much more work is needed in order to understand the process resulting in clinical need in individuals at risk, and the current findings need to be replicated before firm conclusions can be drawn.

\section{From Persistent Subclinical Psychosis to Impairment: Possible Mechanisms}

The positive psychosis pathway may be understood as the phenomenological correlate of the classical dopamine (DA) hypothesis that proposes that hyperactivity of DA transmission from DA cell bodies located in the ventral tegmental area of the midbrain to their terminal fields in the nucleus accumbens and limbic cortex results in the development of positive psychotic symptoms. ${ }^{38,39}$ DAergic hyperactivity may be associated with what has been called a state of endogenous sensitization. ${ }^{38}$ The liability for psychosis may involve deficits in neural regulation resulting in a pathological condition of neurochemical sensitization of the mesolimbic DAergic system analogous to the preclinical model of behavioral sensitization induced by administration of DA-releasing drugs. ${ }^{39}$ Behavioral sensitization is a progressive, enduring enhancement of behaviors that develops following repeated stimulant administration. It is mediated in part by DAergic pathways that are also thought to modulate the development of psychosis. ${ }^{40}$

Endogenous neurochemical sensitization may arise in part as a result of exposure to environmental conditions. ${ }^{41}$ For example, environmental stress can induce sensitization and has been demonstrated to be cross-reactive with many types of pharmacologically induced sensitization, including DA and opiate agonists. ${ }^{39}$ It has been suggested that several environmental risk factors, eg, perinatal injury, childhood trauma, repeated life events, and drug abuse, may contribute to DA sensitization during development. ${ }^{42}$ It is attractive to speculate that a genetictendency to abnormal DA sensitization is associated with the tendency to express low-grade psychotic experiences that in most individuals will only be expressed transitorily over development. However, in the case of developmental exposure to environmental risk factors, individuals may develop a sensitized DA system resulting in abnormally persistent and, eventually, clinically relevant psychotic states. ${ }^{22}$

\section{Methodological Issues}

First, the dynamic hypothesis of psychosis onset was examined in the context of a large-scale epidemiological study not specifically designed for this purpose. It was thus constrained by the specific psychometric tools and sampling frame.

Second, the group comparison between the risk set $(n=845)$ and the nonincluded group $(n=$ 550) showed that the latter had a lower level of education and social status. Because these are risk factors for psychotic experiences, ${ }^{1,10}$ attrition of these individuals may have led to an underestimation of psychosis outcomes in our sample. Bias of reported associations, however, would have occurred only in the unlikely event that high levels of psychosis persistence would be associated with lower rates of transition to clinical psychosis in this group.

Third, in order to measure persistence of psychotic experiences, the SCL-90-R was used. The fact that this instrument only covers the past 2 weeks may have led to an underestimation of the T0-T2 psychosis persistence measure. As it is difficult to see how this would result in a 
spurious association with interviewer-assessed psychotic symptoms and impairment 5 years later, any error resulting from underestimation would have likely been random. Similarly, to the degree that the SCL-90-R overestimates the rate of psychotic symptoms (because of the misunderstandings arising from self-report), any resulting error would likely also be random. Furthermore, the way the measure of psychosis persistence in the present study was constructed, only considering those individuals with the highest $10 \%$ of scores of the continuous SCL-psychosis subscale, prevented misclassification due to overestimation of such experiences on the basis of incidental misunderstanding of items.

Four, the DIA-X/M-CIDI was used to measure clinical relevance of psychotic experiences in terms of psychotic impairment, evaluating secondary dysfunction and help-seeking behavior. On the one hand, help seeking may have led to an underestimation of true positives in the case of individuals who did not seek for help in spite of suffering from true clinical psychotic symptoms. On the other hand, dysfunction may have led to an overestimation of true positives in the case that individuals reported secondary dysfunction only based on subjective level of distress. Nonetheless, the validation of the outcome variable against the clinical judgment of illness strongly supports the validity of psychotic impairment as a proxy measure of clinical relevance.

Five, the number of cases with recurrence-persistence of psychosis expression that developed psychotic impairment was relatively small conform the expected low rate in the general population, resulting in wide CIs.

Six, the main instruments to assess psychotic experiences and symptoms in the current study (SCL-90-R and DIA-X/M-CIDI) are essentially self-report. Although this is likely to have generated false-positive psychotic experiences, likely increasing random error as discussed earlier, there is nevertheless a substantial literature on the (predictive or other forms of) validity of self-reported psychotic symptoms, assessed with DIA-X/M-CIDI, SCL-90-R, and other instruments. ${ }^{2}$ For example, one study validated self-reported psychotic symptoms against a clinical interview with the Brief Psychiatric Rating Scale43 in a sample of psychotic patients, concluding that self-reported scores reflect clinical experience of psychosis. ${ }^{44}$ Poulton et $\mathrm{al}^{14}$ presented strong evidence for predictive validity of CIDI self-reported psychotic symptoms. Similarly, Hanssen et $\mathrm{al}^{45}$ confirmed that the probability of having a psychotic disorder increased in a dose-response fashion with the level of self-reported psychotic experiences in the general population. In 2006, Konings et $\mathrm{al}^{46}$ confirmed the validity and reliability for the measurement of psychotic experiences with a selfreported instrument in the general population. A recent, 20-year longitudinal cohort study on psychotic symptoms using the SCL-90-R also showed high predictive validity of the SCL-90-R psychosis items used in the current sample in terms of deficiencies in social achievement and functioning. ${ }^{33}$

Finally, the current studyhad a longitudinal, populationbased epidemiological perspective, contrasting with the shorter term, selective, sample-enriching approach focusing on help seekers in the ultrahigh-risk literature. ${ }^{36}$ The 2 perspectives and their respective populations, instruments, and outcomes are too different to make findings readily comparable. 


\section{Funding}

This work is further part of the EDSP Study and is supported by the German Federal Ministry of Education and Research (BMBF) project numbers 01EB9405/6, 01EB9910/6, EB10106200, 01EB0140, and 01EB0440. Part of the field work and analyses were also additionally supported by grants of the Deutsche Forschungsgemeinschaft (DFG) LA1148/1-1, WI2246/7-1, and WI709/8-1. European Community's Sixth Framework Programme (Marie Curie Early Stage Research Training Fellowship under contract number MEST-CT-2005-020589 to M.d.G.D.); Dutch Medical Council (VENI grant nr 916.76.147 to M.W.).

\section{Acknowledgments}

Declaration of interest: None. Data access and responsibility: Principal investigators of the EDSP Study are Dr Wittchen and Dr Lieb. Both take responsibility for the integrity of the study data. All authors and coauthors had full access to all study data. Data analysis and manuscript preparation were completed by the authors and coauthors of this article, who take responsibility for its accuracy and content. Core staff members of the EDSP Study group are Dr Kirsten von Sydow, Dr Gabriele Lachner, Dr Axel Perkonigg, and Dr Peter Schuster - Dipl Stat; Michael Höfler, Holger Sonntag, Tanja Brückl, Elzbieta Garczynski, Dr Barbara Isensee, Barbara Spiegel, Andrea Schreier, Dr Ursula Wunderlich, Dr Petra Zimmermann, Datja Beesdo, and Antje Bitner - Dipl Psych; Agnes Nocon and Dr Chris Nelson - Dipl Inf; Hildegard Pfister and Dr Victoria Reed - Dipl Soz. Scientific advisors are Dr Jules Angst (Zurich), Dr Jürgen Margraf (Basel), Dr Günther Esser (Potsdam), Dr Kathleen Merikangas (National Institute of Mental Health, Bethesda), Dr Ron Kessler (Harvard, Boston), and Dr van Os (Maastricht). Financial disclosure: Dr Lieb - speaker honoraria: Wyeth; Dr Wittchen research support: Eli Lilly and Company, Novartis, Pfizer, and Schering-Plough; consulting: Eli Lilly, GlaxoSmithKline Pharmaceuticals, Hoffmann- La Roche Pharmaceuticals, Novartis, Pfizer, and Wyeth; speaker honoraria: Novartis, Schering-Plough, Pfizer, and Wyeth. Dr van Os - past/present unrestricted research support: Eli Lilly, GSK, Organon, BMS, and AstraZeneca; independent speaker honoraria: Eli Lilly, BMS, Lundbeck, Organon, Janssen-Cilag, andAstraZeneca.

\section{References}

1. Johns LC, van Os J. The continuity of psychotic experiences in the general population. Clin Psychol Rev. 2001;21:1125-1141.

2. van Os J, Linscott RJ, Myin-Germeys I, Delespaul P, Krabbendam L. A systematic review and meta-analysis of the psychosis continuum: evidence for a psychosis pronenesspersistence-impairment model of psychotic disorder. Psychol Med. 2009;39:179-195.

3. Angst J, Cassano G. The mood spectrum: improving the diagnosis of bipolar disorder. Bipolar Disord. 2005;7(suppl 4):4-12.

4. Akiskal HS, Vazquez GH. Widening the borders of the bipolar disorder: validation of the concept of bipolar spectrum. Vertex. 2006;17:340-346.

5. Judd LL, Akiskal HS. The prevalence and disability of bipolar spectrum disorders in the US population: re-analysis of the ECA database taking into account subthreshold cases. J Affect Disord. 2003;73:123-131.

6. Constantino JN, Todd RD. Autistic traits in the general population: a twin study. Arch Gen Psychiatry. 2003;60:524-530.

7. Perala J, Suvisaari J, Saarni SI, et al. Lifetime prevalence of psychotic and bipolar I disorders in a general population. Arch Gen Psychiatry. 2007;64:19-28.

8. Chapman LJ, Chapman JP, Kwapil TR, Eckblad M, Zinser MC. Putatively psychosis-prone subjects 10 years later. J Abnorm Psychol. 1994;103:171-183.

9. Stefanis NC, Hanssen M, Smirnis NK, et al. Evidence that three dimensions of psychosis have a distribution in the general population. Psychol Med. 2002;32:347-358.

10. van Os J, Hanssen M, Bijl RV, Ravelli A. Strauss (1969) revisited: a psychosis continuum in the general population? Schizophr Res. 2000;45:11-20. 
11. Yung AR, Phillips LJ, Yuen HP, et al. Psychosis prediction: 12-month follow up of a high-risk (,prodromal“) group. Schizophr Res. 2003;60:21-32.

12. Peters ER, Joseph SA, Garety PA. Measurement of delusional ideation in the normal population: introducing the PDI (Peters et al. Delusions Inventory). Schizophr Bull. 1999;25:553-576. 13. Verdoux H, van Os J, Maurice-Tison S, Gay B, Salamon R, Bourgeois M. Is early adulthood a critical developmental stage for psychosis proneness? A survey of delusional ideation in normal subjects. Schizophr Res. 1998;29:247-254.

14. Poulton R, Caspi A, Moffitt TE, Cannon M, Murray R, Harrington H. Children’s self-reported psychotic symptoms and adult schizophreniform disorder: a 15-year longitudinal study. Arch Gen Psychiatry. 2000;57:1053-1058.

15. Hanssen M, Bak M, Bijl R, Vollebergh W, van Os J. The incidence and outcome of subclinical psychotic experiences in the general population. Br J Clin Psychol. 2005;44(pt 2):

181-191.

16. Kendler KS, Diehl SR. The genetics of schizophrenia: a current, genetic-epidemiologic perspective. Schizophr Bull. 1993; 19:261-285.

17. Spauwen J, Krabbendam L, Lieb R, Wittchen HU, van Os J. Evidence that the outcome of developmental expression of psychosis is worse for adolescents growing up in an urban environment. Psychol Med. 2006;36:407-415.

18. Krabbendam L, Myin-Germeys I, Hanssen M, van Os J. Familial covariation of the subclinical psychosis phenotype and verbal fluency in the general population. Schizophr Res. 2005;74:37-41. 19. Voglmaier MM, Seidman LJ, Niznikiewicz MA, Dickey CC, Shenton ME, McCarley RW. Verbal and nonverbal neuropsychological test performance in subjects with schizotypal personality disorder. Am J Psychiatry. 2000;157:787-793.

20. Stefanis NC, Van Os J, Avramopoulos D, et al. Variation in catechol-o-methyltransferase val158 met genotype associated with schizotypy but not cognition: a population study in 543 young men. Biol Psychiatry. 2004;56:510-515.

21. Fergusson DM, Horwood LJ, Swain-Campbell NR. Cannabis dependence and psychotic symptoms in young people. Psychol Med. 2003;33:15-21.

22. Cougnard A, Marcelis M, Myin-Germeys I, et al. Does normal developmental expression of psychosis combine with environmental risk to cause persistence of psychosis? A psychosis pronenesspersistence model. Psychol Med. 2007;37:513-527.

23. Wittchen HU, Perkonigg A, Lachner G, Nelson CB. Early Developmental Stages of Psychopathology Study (EDSP): objectives and design. Eur Addict Res. 1998;4:18-27.

24. Lieb R, Isensee B, von Sydow K, Wittchen HU. The Early Developmental Stages of Psychopathology Study (EDSP): a methodological update. Eur Addict Res. 2000;6:170-182.

25. Derogatis LR, Cleary PA. Confirmation of the dimensional structure of the SCL-90: a study in construct validation. J Clin Psychol. 1977;33:981-989.

26. Wittchen HU, Pfister H. DIS-X-Interviews: Manual für Screening-Verfahren und Interview; Interviewheft Längsschnittuntersuchung (DIA-X-Lifetime); Ergänzungsheft (DIA-X-Lifetime); Interviewheft Querschnittsuntersuchung (DIA-X-12-Monats-Version); Ergänzungsheft (DIA-X-12 Monats-Version); PC-Programm zur Durchführung der Interviews (Längs- und Querschnittsuntersuchung). Auswertungsprogramm. Frankfurt, Germany: Swets \& Zeitlinger; 1997. 27. World Health Organization. Composite International Diagnostic Interview (CIDI), Version 1.0. Geneva, Switzerland: WHO; 1990.

28. Cottler LB, Helzer JE, Mager D, Spitznagel EL, Compton WM. Agreement between DSM-III and III-R substance use disorders. Drug Alcohol Depend. 1991;29:17-25.

29. Wittchen HU, Robins LN, Cottler LB, Sartorius N, Burke JD, Regier D. Cross-cultural feasibility, reliability and sources of variance of the Composite International Diagnostic Interview (CIDI). The multicentre WHO/ADAMHA field trials. Br J Psychiatry. 1991;159:645-653, 658.

30. Wittchen HU. Reliability and validity studies of the WHO-Composite International Diagnostic Interview (CIDI): a critical review. J Psychiatr Res. 1994;28:57-84.

31. Reed V, Gander F, Pfister H. To what degree does the Composite International Diagnostic Interview (CID) correctly identify DSM-IV disorders? Testing validity issues in a clinical sample. Int J Methods Psychiatr Res. 1998;7:142-155. 
32. Anthony JC, Folstein M, Romanoski AJ, et al. Comparison of the lay Diagnostic Interview Schedule and a standardized psychiatric diagnosis. Experience in eastern Baltimore. Arch Gen Psychiatry. 1985;42:667-675.

33. Rossler W, Riecher-Rossler A, Angst J, et al. Psychotic experiences in the general population: a twenty-year prospective community study. Schizophr Res. 2007;92:1-14.

34. Henquet C, Krabbendam L, Spauwen J, et al. Prospective cohort study of cannabis use, predisposition for psychosis, and psychotic symptoms in young people. BMJ. 2005;330:11.

35. Escher S, Romme M, Buiks A, Delespaul P, Van Os J. Independent course of childhood auditory hallucinations: a sequential 3-year follow-up study. Br J Psychiatry. 2002;43:s10-s18.

36. Van Os J, Delespaul P. Toward a world consensus on prevention of schizophrenia. Dialogues Clin Neurosci. 2005;7:53-67.

37. Dominguez MDG, Viechtbauer W, Simons CJP, Van Os J, Krabbendam L. Are psychotic psychopathology and neurocognition orthogonal? A systematic review of their associations. Psychol Bull. 2009;135:157-171.

38. Laruelle $\mathrm{M}$. The role of endogenous sensitization in the pathophysiology of schizophrenia: implications from recent brain imaging studies. Brain Res Brain Res Rev. 2000;31:371-384.

39. Lieberman JA, Sheitman BB, Kinon BJ. Neurochemical sensitization in the pathophysiology of schizophrenia: deficits and dysfunction in neuronal regulation and plasticity.

Neuropsychopharmacology. 1997;17:205-229.

40. Wolf ME, White FJ, Nassar R, Brooderson RJ, Khansa MR. Differential development of autoreceptor subsensitivity and enhanced dopamine release during amphetamine sensitization. $\mathrm{J}$ Pharmacol Exp Ther. 1993;264:249-255.

41. Laruelle M, Kegeles LS, Abi-Dargham A. Glutamate, dopamine, and schizophrenia: from pathophysiology to treatment. Ann N Y Acad Sci. 2003;1003:138-158.

42. Murray RM, McDonald C, Bramon E. Neurodevelopmental impairment, dopamine sensitisation, and social adversity in schizophrenia. World Psychiatry. 2002;1:137-145.

43. Overall JE, Gorham DR. The brief psychiatric rating scale. Psychol Rep. 1962;10:799-812.

44. van Os J, Verdoux H, Maurice-Tison S, et al. Self-reported psychosis-like symptoms and the continuum of psychosis. Soc Psychiatry Psychiatr Epidemiol. 1999;34:459-463.

45. Hanssen MS, Bijl RV, Vollebergh W, van Os J. Self-reported psychotic experiences in the general population: a valid screening tool for DSM-III-R psychotic disorders? Acta Psychiatr Scand. 2003;107:369-377.

46. Konings M, Bak M, Hanssen M, van Os J, Krabbendam L. Validity and reliability of the CAPE: a self-report instrument for the measurement of psychotic experiences in the general population. Acta Psychiatr Scand. 2006;114:55-61. 